\title{
„Ambulant vor stationär“
}

\section{Theoretische Anreizanalysen und empirische Ergebnisse zur Prüfung eines gesundheitspolitischen Grundsatzes}

\author{
R. Seitz, F-G. Krammling, C. Lindl
}

Das GKV-Modernisierungsgesetz (GMG) hat erstmals für das Jahr 2004 die Datengrundlage für gezielte Vertragsanalysen der Krankenkassen im vertragsärztlichen Sektor geschaffen. Mit einer Gruppe repräsentativer Betriebskrankenkassen nutzte der BKK Landesverband Bayern die neue Datenbasis für die empirische Prüfung des Grundsatzes „ambulant vor stationär". Dazu wurde die Entwicklung stationsersetzender Leistungen sowohl im strukturvertraglich geförderten ambulant-vertragsärztlichen als auch im Krankenhaussektor analysiert. Diese empirische Untersuchung steht im Mittelpunkt des Beitrags. Vorgelagert sind eine theoretische Anreizanalyse aus der Perspektive der gesetzlichen Krankenversicherung und die Beschreibung der Ausgabenentwicklung für den ambulanten und stationären Bereich auf GKV-Ebene seit dem Jahr 1991. Die empirische Studie ist ein Prä-Post-Vergleich für die Jahre 2004, 2005 und 2006. Sie bezieht sich auf sieben prospektiv ausgewählte Leistungsbereiche (Operationen Leisten- und Schenkelhernie, Katarakt, Varizen, Arthroskopie, Karpaltunnelsyndrom, Strahlentherapie und kurative Koloskopien) und umfasst für das Jahr 2006 über 40.000 Leistungen. Primäre Zielgröße ist die Entwicklung von Fallzahlen. Die unterschiedliche Kostenintensität der Leistungen wird durch eine DRG-Gewichtung berücksichtigt. Als Ergebnis zeigt sich eine Fallzahlsteigerung sowohl im vertragsärztlichambulanten als auch im stationären Bereich. Diese liegt kostengewichtet pro Jahr durchschnittlich bei 8,2\%, und ist primär auf einen massiven Fallzahlanstieg im Krankenhaussektor zurückzuführen. Trotz Unschärfen und Einschränkungen hinsichtlich Methodik und Datenbasis weist das Ergebnis darauf hin, dass es in den betrachteten Jahren nicht gelungen ist, den Grundsatz „ambulant vor stationär“ mit dem Ziel der Beitragssatzstabilität konsequent umzusetzen. ${ }^{1}$

In den vielen Gesundheitsreformen der letzten Jahrzehnte blieb ein gesundheitspolitischer Grundsatz unangetastet: der Grundsatz „ambulant vor stationär“, verankert in $\S 73$ Abs. 4 des Fünften Buchs Sozialgesetzbuch (SGB V). „Krankenhausbehandlung darf nur verordnet werden, wenn eine ambulante Versorgung der Versicherten zur Erzielung des Heil- oder Linderungserfolgs nicht ausreicht", lautet die Vorschrift für die Haus- und Fachärzte. Das leistungsrechtliche Pendant dieser Vorgabe findet sich in § 39 Abs. 1 SGB V, wonach der Versicherte nur dann Anspruch auf stationäre Krankenhausbehandlung hat, wenn ambulante Versorgungsformen im Hinblick auf das Behandlungsziel nicht ausreichen. Freilich ist die praktische Umsetzung dieser Normen häufig schwierig. Seit Jahren dienen jedenfalls vermutete Einsparungen in der stationären Krankenhausbehandlung als Begründung für neue Leistungen, Verträge und Projekte im ambulanten

Dr. rer. pol. Robert Seitz, BKK Landesverband Bayern, München, Abteilungsleiter Ambulante Versorgung Dr. med. Franz-Georg Krammling, BKK Landesverband Bayern, München, Stabstelle Medizinische Grundsatzfragen

Dr. rer. pol. Christian Lindl, BKK Landesverband Bayern, München, Abteilungsleiter Stationäre Versorgung, Pflegeversicherung
Sektor. Ob durch die entsprechenden Anreize jeweils Verlagerungseffekte tatsächlich eintreten, wurde dagegen kaum untersucht, zumindest nicht öffentlich transparent gemacht. Im vorliegenden Beitrag soll eine inhaltliche und empirische Prüfung des Grundsatzes „ambulant vor stationär“ am Beispiel gesamtvertraglich geförderter „stationsersetzender Leistungen " vorgenommen werden.

\section{Anreizanalyse zum ambulanten und stationären Sektor}

Leistungsspektrum zwischen ambulant und stationär

Wichtigste Träger der ambulanten Versorgung sind die niedergelassenen Haus- und Fachärzte. Für die gesetzliche Krankenversicherung (GKV) haben die 17 regionalen Kassenärztlichen Vereinigungen (KVen) den „Sicherstellungsauftrag“, der eine flächendeckende, wohnortnahe ambulante Versorgung gewährleisten soll. Eine relativ neue, mit dem GKV-Modernisierungsgesetz (GMG) eingeführte, ambulante Versorgungsform sind die fachübergreifenden medizinischen Versorgungszentren (MVZ). In einem MVZ arbeiten Ärzte oft als Angestellte - ein Beschäftigungsverhältnis, das seit der Flexibilisierung des Vertragsarztrechts 


\section{THEMA}

auch bei niedergelassenen Ärzten deutlich erleichtert wurde. MVZ können auch an einem Krankenhaus angesiedelt sein.

Krankenhausärzte oder spezialisierte Einrichtungen können darüber hinaus bei entsprechendem Bedarf für die vertragsärztliche Versorgung ermächtigt werden. Zugelassene Ärzte (auch: Psychotherapeuten), zugelassene MVZ und ermächtigte Ärzte sowie ermächtigte ärztlich geleitete Einrichtungen nehmen an der vertragsärztlichen Versorgung teil.

Zum ambulanten Spektrum gehören auch folgende ärztliche Leistungen, die ambulant im bzw. am Krankenhaus angeboten werden:

- Leistungen der Hochschulambulanzen und psychiatrischen Institutsambulanzen ( $§ 117$ ff SGB V),

- hochspezialisierte Leistungen zur Behandlung seltener Erkrankungen und von Erkrankungen mit besonderen Krankheitsverläufen (§ 116b SGB V),

- ambulantes Operieren ( $(115 b$ SGB V),

- vor- und nachstationäre Behandlung (§ 115a SGB V).

Kennzeichnend für die stationäre Versorgung ist, dass eine Institution mit Unterkunft und Verpflegung - in der Regel über Nacht - für die Behandlung notwendig ist. Neben angestellten Ärzten können auch Vertragsärzte als Belegärzte stationäre oder teilstationäre Leistungen im Krankenhaus erbringen. Die ärztlichen Leistungskomponenten belegärztlicher Leistungen selbst werden aus der vertragsärztlichen Gesamtvergütung honoriert.

Mit der integrierten Versorgung (\$§ 140a ff SGB V) hat der Gesetzgeber eine Basis für selektivvertraglich zu vereinbarende innovative Versorgungsformen geschaffen, die eine flexible Zuordnung von Patienten gemäß ihrem Versorgungsbedarf an der Nahtstelle von ambulantem und stationärem Bereich erleichtern sollen. In der Versorgungspraxis werden jedoch Mängel bei der inhaltlichen Umsetzung der Integration konstatiert. Die bislang abgeschlossenen Verträge zu §§ 140a ff zielen offenbar weniger auf die Integration $^{2}$ als auf die in diesen Paragraphen ermöglichte Anschubfinanzierung. Damit können die projektbezogenen Leistungsausgaben der Krankenkassen durch Kürzungen in anderen Bereichen gegenfinanziert werden. Zur validen Beurteilung der neuen Versorgungsformen wäre eine unabhängige Evaluation erforderlich. Diese ist jedoch gesetzlich nicht vorgesehen. Ergebnisse freiwilliger wissenschaftlicher Evaluationsstudien stehen bislang aus - trotz einer umfassenden Beschreibung innovativer Projekte und Konzepte (Weatherly et al. 2007). Die Bewertung wird erheblich dadurch erschwert, dass es im Bereich der neuen Versorgungsformen wenig Transparenz und bislang auch keine Rahmenvorgaben für den Datenfluss und Datenaustausch gibt.

Eine nicht nur von vielen Gesundheitssystemforschern geforderte, sondern auch gesetzlich vorgesehene Option zur Schnittstellenoptimierung stellen Einrichtungen dar, in denen die Patienten durch Zusammenarbeit mehrerer niedergelassener Ärzte ambulant und stationär versorgt werden können (Praxiskliniken). Obwohl der Gesetzgeber schon 1989 Krankenkassen, KVen und Landeskrankenhausgesellschaften verpflichtet hat, durch dreiseitige Verträge die Behandlung in Praxiskliniken zu fördern ( $\$ 115$ Abs. 2 SGB V), ist dies bis heute nicht umgesetzt worden. Dabei hat auch keiner der genannten Vertragspartner von der Möglichkeit zur Anrufung der Landesschiedsstelle bei Blockade einer Seite Gebrauch gemacht. Ebenso wenig sind die Landesregierungen ihrer Verpflichtung nach § 115 Abs. 4 SGB V nachgekommen, den Inhalt der dreiseitigen Verträge durch Rechtsverordnung zu bestimmen (Schulte 2007).

\section{Anreize von Krankenkassen und Leistungserbringern}

Die Bewertung einer Fragestellung (z. B. ambulant vor stationär)? hängt von der Perspektive des Betrachters ab. Aus Perspektive der GKV sind auf der Kostenseite die Ausgaben der jeweiligen Krankenkassen maßgeblich.

Vergütung ambulant-ärztlicher Leistungen innerhalb der pauschalierten Gesamtvergütung

Erfolgt eine Leistung im ambulant-ärztlichen Sektor, so fließt die Vergütung der Krankenkasse über die KV an die Ärzte. Die klassische Vergütungsform ist die Kopfpauschale: Eine Krankenkasse zahlt pro Mitglied bzw. Rentner einen festen $€$-Betrag „mit befreiender Wirkung“. Damit sind alle tatsächlich anfallenden Leistungen abgegolten. Werden durch Vertragsärzte mehr ambulante Behandlungsleistungen erbracht, sinkt der entsprechende Punktwert für diese Leistungen. Die Krankenkasse hat deshalb bei einer zusätzlichen Leistung keine zusätzlichen Kosten. Wenn Leistungen aus dem stationären in den ambulanten Bereich verlagert werden, entstehen für die Krankenkasse somit keine Zusatzausgaben. Der Anreiz für die Krankenkassen hinsichtlich der Leistungserbringung ist eindeutig: ambulant vor stationär, da im stationären Bereich dem Krankenhaus anfallende Behandlungskosten jeweils direkt zu vergüten sind.

\section{Vergütung außerhalb der pauschalierten Gesamtvergütung}

Eine Reihe von Leistungen wird entweder durch Vorgaben des Gesetzgebers oder Strukturverträge mit der KV außerbudgetär vergütet, $d$. h. mit festen Pauschalen oder Punktwerten. Eine Zunahme von Behandlungsleistungen führen damit zu zusätzlichen Ausgaben der Krankenkassen bzw. zusätzlichen Einnahmen der Vertragsärzte. Für Betriebskrankenkassen liegt der Anteil in Bayern insgesamt bei ca. $40 \%$ der Gesamtvergütung, wobei etwa die Hälfte auf Strukturverträge entfällt. Die Vergütungssätze sind dabei i. a. höher als die „floatenden“ Punktwerte innerhalb der Pauschalvergütung. Auch für viele krankenhausersetzende Leistungen erfolgt die Vergütung extrabudgetär. Dies bietet niedergelassenen Ärzten den Anreiz, solche Leistungen - z. B. Operationen oder aufwändige internisti- 
sche Leistungen - selbst ambulant durchzuführen und die Patienten dafür nicht ins Krankenhaus zu überweisen.

\section{Unterschiedliche Finanzierung bei Kapitalkosten}

Kapitalkosten werden im akutstationären Bereich - wenn auch mit abnehmender Tendenz - von den Bundesländern aus Steuermitteln getragen, während die laufenden Betriebskosten durch die Krankenkassen bestritten werden (sog. duale Finanzierung). Eine Gruppe von Gesundheitsökonomen ${ }^{3}$ hat im Jahr 1999 den Anteil der Kapitalkosten an den gesamten Nutzerkosten auf $18 \%$ bis $26 \%$ geschätzt; in den letzten Jahren ist dieser Anteil wegen der abnehmenden Investitionsneigung der Bundesländer jedoch zurückgegangen. Der Gesetzgeber berücksichtigt grundsätzlich die öffentliche Förderung von Krankenhäusern, z.B. durch einen in § 120 Abs. 3 SGB V verankerten Investitionskostenabschlag von 10\% bei der Vergütung von Hochschulambulanzen, psychiatrischen Institutsambulanzen, sozialpädiatrischen Zentren und sonstigen ermächtigten ärztlich geleiteten Einrichtungen. Im ambulant-vertragsärztlichen Bereich müssen die Kapitalkosten vollständig aus der Vergütung durch die Krankenkassen bestritten werden.

\section{Zuordnung von Arzneimitteln, Heil- und Hilfsmitteln und weite- ren assoziierten Leistungsarten}

Beim Vergleich stationär - ambulant ist die Vergütungssystematik der unterschiedlichen Leistungsarten zu beachten: Während in der stationären Vergütung sowohl ärztliche Leistungen als auch die Arzneimittel-Abgabe wie auch weitere Ausgabenbereiche, z. B. Krankengymnastik und stationär eingesetzte Hilfsmittel, enthalten sind, erfolgt im ambulanten Bereich ein getrennter Ausweis der Leistungsarten. Mit der Krankenhausvergütung sind auch sog. „unechte“ Gemeinkosten (personenbezogen verwendete Verbrauchsmaterialien wie Spritzen, Verbände, Bandagen) abgedeckt. Im ambulanten Bereich werden diese im Rahmen des sog. „Sprechstundenbedarfs“ pauschal und nicht patientenbezogen abgerechnet. Bei einem exakten Vergleich des Versorgungsaufwands in den beiden Sektoren muss neben dem medizinischen auch der nichtmedizinische Ressourcenverbrauch berücksichtigt werden, beispielsweise für Unterkunft und Verpflegung sowie für häusliche Krankenpflege und für Haushaltshilfe.

\section{Vergütungssystem im Krankenhaus}

Bei der Vergütung von Krankenhäusern nach tagesgleichen Pflegesätzen haben die Kostenträger den finanziellen Anreiz, auf eine möglichst frühzeitige Entlassung in den ambulanten Bereich hinzuwirken, da dort die Behandlungskosten pro Tag im Allgemeinen niedriger sind als der Krankenhauspflegesatz. Seit 2004 ist diese Vergütungsform im Wesentlichen auf Psychiatrie und Psychosomatik beschränkt. Ansonsten werden diagnose- bezogene Fallpauschalen (DRGs) abgerechnet - mit einer anderen Anreizwirkung: Nun ist es im Prinzip für eine Krankenkasse finanziell vorteilhaft, wenn aufwändige Fälle stationär aufgenommen werden, die dann möglichst lange im Krankenhaus verbleiben sollen. ${ }^{4}$ Bei leichten Fällen streben die Krankenkassen einen Aufenthalt unterhalb der unteren Grenzverweildauer an, da dies Abschläge für die Krankenhausabrechnung bedeutet. Erst bei Überschreiten der oberen Grenzverweildauer einer DRG stellt sich die Frage nach den Mehrkosten gegenüber der ambulanten Weiterversorgung. Die Krankenhäuser haben als Leistungserbringer bei den DGRs den Anreiz, möglichst viele Fälle zu produzieren bzw. einen Krankenhausfall mit mehreren Diagnosen zu splitten, damit mehrere DRGs abgerechnet werden können. ${ }^{5}$

Neben den DRGs sind für einen Kostenvergleich mit dem ambulanten Bereich, soweit für die jeweilige Leistung relevant, auch Zusatzentgelte zu berücksichtigen. Diese sind für bestimmte sehr aufwändige Verfahren und sehr teure Arzneimittel bzw. Blut- und Plasmaprodukte vorgesehen. Bei belegärztlichen Leistungen fällt neben den DRGs, die in diesem Falle keine ärztlichen Leistungen enthalten, die vertragsärztliche außerbudgetäre Vergütung für Belegärzte (ggf. auch Anästhesisten) an. Ambulante Operationen am Krankenhaus (gem. § 115b SGB V) werden nach festen, auf Landesebene vereinbarten Punktwerten vergütet. Diese orientieren sich an der entsprechenden Vergütung niedergelassener Fachärzte und sollen die Gesamtkosten einschließlich Kapitalkosten abdecken. Gleichwohl haben die Krankenhäuser den Anreiz, bei der Leistungserbringung auf bestehende stationäre Infrastruktur zurückzugreifen, die aus Steuermitteln oder DRGs finanzierte wurde.

Die Krankenhäuser haben das Recht, prinzipiell ambulant zu versorgende Patienten stationär aufzunehmen, soweit dies durch Ko-Morbiditäten oder Komplikationen begründet werden kann. Die Prüfung der Notwendigkeit der stationären Aufnahme in solchen Fällen stellt die Kostenträger bzw. den Medizinischen Dienst der Krankenkassen vor eine große Herausforderung.

\section{Perspektive anderer Kostenträger}

Besonders komplex ist die Anreizanalyse, wenn weitere Kostenträger berücksichtigt werden. Beispielhaft sei dies am Bereich Rehabilitation erläutert. Wenn die Behandlung eines Versicherten mit eingeschränkter Erwerbsfähigkeit im häuslichen Umfeld des Patienten möglich ist, dürfte dies mit Blick auf die Gesamtkosten (alle Kostenträger) kostengünstiger sein als eine stationäre, wohnortferne mehrwöchige Reha-Maßnahme. Die ambulante medizinische Versorgung fällt aber im Allgemeinen in die Trägerschaft der Krankenkasse, während die Rehabilitation zur Wiederherstellung der Erwerbsfähigkeit durch die Rentenversicherungsträger finanziert wird. Da diese mit dem sog. Übergangsgeld auch für Lohnersatzleistungen aufkommen, während die Krankenkassen Krankengeld zahlen müssten, haben letztere einen klaren finanziellen Anreiz, die Kosten auf die Rentenversicherungsträger zu verlagern. 
In wissenschaftlichen Studien wurde die stationäre mit der ambulanten Rehabilitation verglichen. Tendenziell wurde bei der Kostenbetrachtung aus einer gesellschaftlichen Perspektive oder aus Sicht der Rehabilitationsträger eine Überlegenheit der ambulanten Rehabilitation festgestellt. Deshalb wird ein weiterer Ausbau dieses Bereichs angestrebt und erwartet (z. B. Krauth et al. 2005b).

\section{Empirische Prüfung des Grundsatzes}

Für den empirischen Vergleich des ambulanten mit dem stationären Sektor im deutschen Gesundheitswesen gibt es zwei Ansatzpunkte: (1) die „Vogelperspektive“ der Ausgabenentwicklung in der gesetzlichen Krankenversicherung insgesamt und (2) die Betrachtung auf Niveau des Individuums (d. h. des einzelnen Patienten bzw. Versicherten) im Rahmen gezielter Studien.

\section{Die Vogelperspektive: Globale Ausgabenentwicklung in den Leistungssektoren}

Die amtliche Statistik für die GKV weist die nominale Ausgabenentwicklung für die verschiedenen Leistungsbereiche getrennt aus. Abbildung 1 zeigt diese Entwicklung seit dem Jahr 1991 (Index = 100, ohne Diskontierung oder Berücksichtigung von Inflation) mitgliedsbezogen für die Bereiche ambulante ärztliche Behandlung, Arzneimittel, Heil- und Hilfsmittel und Krankenhaus. Auch die Entwicklung der GKV insgesamt ist aufgeführt. Da aus der Indexdarstellung nicht die absoluten GKV-Ausgaben für einen
Bereich hervorgehen, sind zusätzlich diese Werte für 2006 in Mrd. € angeführt.

Die niedrigste Steigerungsrate pro Mitglied (44\%) weist im Berichtszeitraum die ambulant-ärztliche Behandlung auf. Im Krankenhaussektor liegt die Steigerungsrate je Mitglied mit 66\% trotz einer starken Verkürzung der durchschnittlichen Verweildauer von über einem Drittel und einem starken Rückgang der Bettenzahl ${ }^{6}$ um fast ein Viertel (Statistisches Bundesamt 2006) über dem GKVDurchschnitt (55\%). Noch höher ist - wegen der jüngsten Entwicklung - der Ausgabenanstieg für den Bereich der ambulant verabreichten Arzneimittel mit einer Steigerung von $76 \%$ seit 1991 .

Diese makroökonomischen Ergebnisse bieten keine Anhaltspunkte dafür, dass es in Deutschland in den letzten 15 Jahren gelungen ist, den Grundsatz „ambulant vor stationär" umzusetzen. Die Zeitreihen-Analyse anhand hoch aggregierter Daten ist jedoch geprägt von den politischen und rechtlichen Rahmenbedingungen im betrachteten Zeitraum und den damit verbundenen Anreizwirkungen auf die verschiedenen Akteure. Eine valide Schlussfolgerung ist deshalb nur sehr eingeschränkt zulässig.

Die Krankenkassen können ihre eigenen Ausgaben in den unterschiedlichen Leistungsbereichen den standardisierten, aus dem Risikostrukturausgleich (RSA) bundeseinheitlich zugewiesenen Leistungsausgaben gegenüberstellen. Bei einigen bayerischen Betriebskrankenkassen liegen die tatsächlichen Ausgaben für Behandlung im Krankenhaus niedriger als die RSA-Standardwerte, für ärztliche Behandlung sind dagegen die tatsächlichen Ausgaben höher.

\section{Abbildung 1: Ausgabenentwicklung GKV 1991 bis 2006 (je Mitglied)}

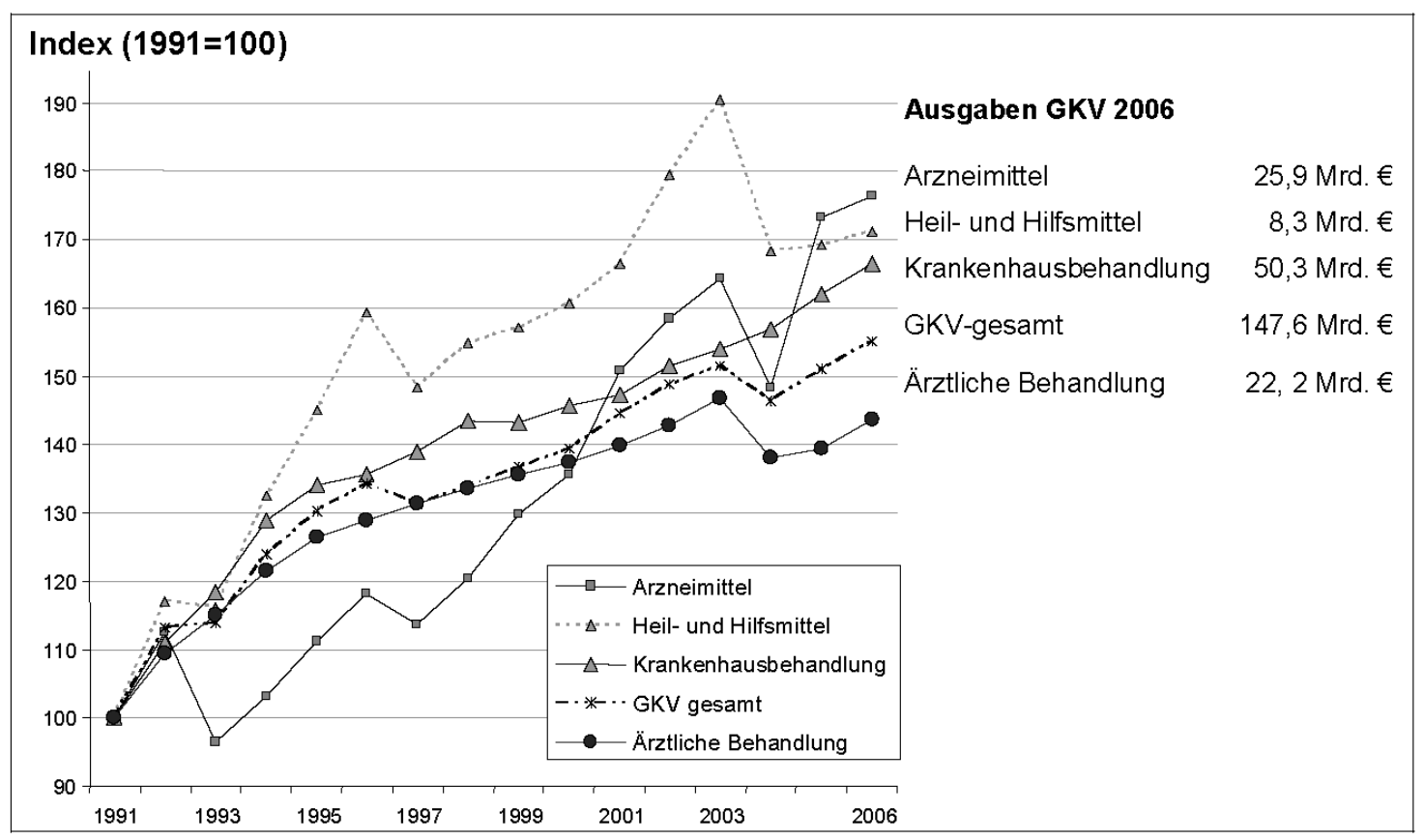

Quelle: KJ 1-Statistik 1991-2006, Bundesgesundheitsministerium, eigene Berechnungen 
Dies könnte als Hinweis für die Umsetzung des Grundsatzes „ambulant vor stationär“ in Bayern interpretiert werden. Allerdings ist der Abstand zwischen den tatsächlichen Krankenhausausgaben und den RSA-Zuweisungen in den letzten Jahren zurückgegangen.

Aus den Durchschnittswerten ist kein Rückschluss auf die tatsächliche Leistungsmenge sowie deren Vergütung und Entwicklung möglich. Fundierte Aussagen über die Leistungsentwicklung in den verschiedenen Versorgungssektoren lassen sich nur im Rahmen von gezielten Studien mit versichertenbezogenen Daten gewinnen.

\section{Empirische Untersuchung im Rahmen von Studien - ein Beispiel aus der Vertragspraxis zu stationser- setzenden Leistungen}

Der BKK Landesverband Bayern hat mit der KV Bayerns zur Förderung fachinternistischer Leistungen und ambulanter Operationen im Rahmen des Gesamtvertrags ab 1998 Strukturverträge abgeschlossen, die eine außerbudgetäre Vergütung auf der Grundlage von Einzelleistungen vorsehen. Dazu ist der Betrag des Ausgabenvolumens (Obergrenze) festzulegen sowie eine Regelung zur Vermeidung der Überschreitung dieses Betrages zu treffen ( $\$ 85$ Abs. 2 SGB V). Als Regelung zur Vermeidung der Überschreitung der Obergrenze wurde vertraglich festgehalten, dass die Vertragspartner prospektiv und zeitnah Kriterien zur Beurteilung einer Mengenentwicklung vereinbaren. Für die gesamtvertragliche Honorarvereinbarung des BKK Landesverband Bayern für das Jahr 2005 betrug beispielhaft die Obergrenze für strukturvertragliche Leistungen 132 Mio. €. Gemäß dem Grundsatz der Beitragssatzstabilität (§ 71 SGB V) steigt diese Obergrenze für das Jahr 2006 entprechend der Entwicklung der beitragspflichtigen Einnahmen (Grundlohnsumme). Diese Rate wird durch das Bundesgesundheitsministerium als Richtschnur für die Vertragsverhandlungen des Folgejahres bekannt gegeben. Sie liegt seit vielen Jahren unter einem Prozent.

Da, insbesondere infolge des GKV-Modernisierungsgesetzes (GMG), erst ab dem Leistungsjahr 2004 die Datenlage für versichertenbezogene Auswertungen im vertragsärztlichen Bereich geschaffen wurde, können die Krankenkassen erst seitdem selbst entsprechende Auswertungen durchführen. Somit konnten erstmals die Leistungs- und Vertragszeiträume ab 2004 verglichen werden.

Als Studientyp zur Überprüfung der Fragestellung kam nur der Vorher-Nachher-Vergleich infrage. Dadurch ergeben sich mögliche Verzerrungen und Unschärfen, die beispielsweise auf die Änderung folgender externer Rahmenbedingungen in den untersuchten Jahren zurückzuführen sind:

- die Einführung einer neuen Gebührenordnung für Vertragsärzte (EBM 2000plus) zum 01.04.2005

- die Umstellung der Krankenhausabrechnung auf DRGs und die jährlichen Änderungen der DRG-Systems (um- fasst auch Anpassungen der ICD und OPS, der Diagnoseund Prozedurenkodierung)

- die schrittweise Etablierung der Teilnahme der Krankenhäuser am elektronischen Datenträgeraustausch (§ 301 SGB V).

Folgende Basisannahmen liegen der Untersuchung zugrunde:

- Die niedergelassenen Ärzte nehmen eine Lotsenfunktion bei der Versorgungssteuerung in den stationären Bereich wahr.

- Als Referenz und Benchmark der Bewertung für das Versorgungsniveau eines Jahres (Leistungsmenge insgesamt) gilt das Niveau des Vorjahres.

- Primäres Aufgreifkriterium für den Leistungsbereich ist die jeweilige EBM- Ziffer bzw. der zugrunde liegende OPS- Code.

- Die Leistungsmenge wird als Surrogatparameter für die Ausgabenwirkung betrachtet. ${ }^{7}$ Zur Abbildung der unterschiedlichen Kostenintensität der einzelnen Leistungen wird deren relative Gewichtung im DRG-System (Mittelwert der Relativgewichte für die einzelnen Prozeduren) herangezogen.

\section{Fragestellung}

Eine Verlagerung aus dem stationären in den ambulanten Bereich wird durch die Krankenkassen grundsätzlich dann akzeptiert, wenn die Gesamtheit der Leistungen pro Mitglied von einem Jahr auf das nächste insgesamt die Vergleichsgröße für das Vorjahr nicht übersteigt. ${ }^{8}$ Zur Bestimmung der Leistungsmenge wird für jedes Jahr die Summe aus (a) vertragsärztlich-ambulanten, (b) ambulant am Krankenhaus erbrachten (ambulantes Operieren gem. § 115b SGB V) sowie stationär im Krankenhaus in (c) Haupt- und (d) Belegabteilungen erbrachten Leistungen gebildet.

\section{Umsetzung durch den BKK Landesverband und Betriebskrankenkassen}

Der BKK Landesverband Bayern erstellte die Basiskriterien zur Beurteilung der Obergrenzen-Überschreitung und stimmte diese mit der KV Bayerns ab. Die Kriterien beziehen sich auf die Fragestellung, die Auswertungsmethode, die beteiligten Betriebskrankenkassen und die ausgewählten Leistungsbereiche. Damit wurde das Evaluationskonzept prospektiv festgelegt. Das Analyseprojekt wurde zusammen mit ausgewählten Betriebskrankenkassen durchgeführt, die Mitglied der Arbeitsgruppe Vertragsärztliche Vergütung beim BKK Landesverband sind: Audi BKK, BKK BMW, BKK Mobil Oil, HypoVereinsbank BKK, Persönliche BKK (PBK) sowie Siemens Betriebskrankenkasse (SBK). Diese Krankenkassen können als repräsentativ für das BKK System betrachtet werden. Auf sie entfallen ca. $40 \%$ der BKK Mitglieder in Bayern. Sie weisen eine kon- 
Tabelle 1: Verlagerungseffekte ohne Kostengewichtung (Fallzahlen)

\begin{tabular}{|l|c|c|c|c|}
\hline & $\mathbf{2 0 0 4}$ & $\mathbf{2 0 0 5}$ & $\mathbf{2 0 0 6}$ & Anstieg p. a. \\
\hline stationär Hauptabteilung & 4.922 & 4.788 & 6.674 & $16,4 \%$ \\
\hline stationär Belegabteilung & 1.959 & 1.715 & 1.646 & $-8,3 \%$ \\
\hline ambulant am Krankenhaus & 2.403 & 2.879 & 3.428 & $19,4 \%$ \\
\hline vertragsärztlich ambulant & 26.851 & 27.008 & 28.085 & $2,3 \%$ \\
\hline SUMME & $\mathbf{3 6 . 1 3 5}$ & $\mathbf{3 6 . 3 8 9}$ & $\mathbf{3 9 . 8 3 3}$ & $\mathbf{5 , 0} \%$ \\
\hline
\end{tabular}

stante Mitgliederzahl im Vergleichszeitraum auf. In der Summe war die Mitgliederzahl der betrachteten Krankenkassen zwischen 2004 und 2005 konstant (Veränderung 0,0\%), bis 2006 wuchs sie um 1,72\% (Quelle: Amtliche Statistik KM 6 zum Stichtag 01.07. des Jahres). Zur technischen Umsetzung wurden für den vertragsärztlich-ambulanten Bereich und den Krankenhausbereich Abfragen mittels InfoNet (Datawarehauslösung des BKK-Systems als primäres Auswertungsinstrument) und ISKV (umfassende Informationssysteme von Betriebs-, Innungs- und Ersatzkassen zur Validierung der Krankenhausdaten) entwickelt. Zur Ermittlung der Fallzahlen für ambulantes Operieren am Krankenhaus wurde zusätzlich auf die Abrechnungssoftware KAPS zurückgegriffen.

\section{Leistungsbereiche}

Bei der Auswahl der Leistungsbereiche lagen folgende Kriterien zugrunde: (1) Repräsentativität für die Strukturverträge des BKK Landesverband Bayern mit der KV Bayerns; (2) Potenzial zur Verlagerung aus dem stationären in den ambulanten Bereich; (3) datentechnisch und inhaltlich gute Abgrenzbarkeit der Leistungen. Auf dieser Basis wurde folgende Auswahl getroffen: Operation Leisten- und Schenkelhernie, kurative Koloskopien (Dickdarmspiegelungen), Strahlentherapie, Kataraktoperation (Einbringen einer neuen Linse bei grauem Star), rekonstruktive arthroskopische Operationen (bei schwereren Knie- wie Schultergelenkverletzungen), Operation von Varizen (Krampfadern) und Karpaltunnel- sowie Tarsaltunnelsyndrom (Nervenstörung im Bereich der Handwurzel bzw. der Fußwurzel).

\section{Ergebnisse}

Für die untersuchten Leistungsbereiche wurden die in den Tabellen dargestellten Fallzahlen ermittelt. In den Tabellen 1 und 2 erfolgt die summarische Darstellung der Ergebnisse über den gesamten Betrachtungszeitraum 2004 bis 2006. Alle Zahlen sind jeweils um die Mitgliederentwicklung bereinigt. Wenn eine Krankenkasse ihre Mitgliederzahl beispielsweise im Vergleich zum Vorjahr verdoppelt, wirkt sich dies in der Analyse als Halbierung der Fallzahlen aus.

Während in Tabelle 1 die Fallzahlen ohne Kostengewichtung über die betrachteten Leistungsbereiche aufsummiert werden, berücksichtigt die Summenbildung

in Tabelle 2 die unterschiedliche Kostenintensität der Leistungen.Die angegeben Veränderungsraten in der rechten Spalte beziehen sich auf den durchschnittlichen Anstieg pro Jahr zwischen 2004 und $2006 .^{9}$

Als Hauptergebnis zeigt sich ein massiver Anstieg der Fallzahlen im Krankenhausbereich. Die stationären Hauptabteilungen weisen diesen Anstieg erst ab dem Jahr 2005 auf, die ambulanten Operationen am Krankenhaus steigen über den gesamten Betrachtungszeitraum. Im vertragsärztlich-ambulanten Bereich beträgt der Anstieg jährlich über zwei Prozent. Er ist somit höher als der Anstieg der beitragspflichtigen Einnahmen zur GKV. Im Krankenhaus-Bereich, für den der Grundsatz der Beitragssatzstabilität ebenfalls gilt, liegt der Anstieg der Fallzahlen bei 12,5\% pro Jahr und somit weit über der Steigerungsrate der GKV-Einnahmen.

In der ungewichteten Summenbildung in Tabelle 1 wird jede Operation als ein Fall gezählt - unabhängig davon, ob die Prozedur aufwändig (z. B. Strahlentherapie) oder weniger aufwändig (z. B. Koloskopie) ist. In Tabelle 2 sind die Ergebnisse kostengewichtet wiedergegeben. Dazu wurde jeder Fall mit dem DRG-Relativgewicht multipliziert, so dass z. B. das Gewicht einer Strahlentherapie rund fünf Mal so hoch ist wie das einer kurativen Koloskopie.

Nach Kostengewichtung der Leistungsbereiche fällt der jährliche Anstieg der Fallzahlen insgesamt noch stärker aus. Im Vergleich zum Vorjahr liegt er 2005 bei 3,5\% und 2006 bei 13,6\%. Auch hier ist der Anstieg der Krankenhausfallzahlen mit jährlich 13,9\% im Durchschnitt mehr als doppelt so hoch wie der Vergleichswert im ambulantvertragsärztlichen Bereich.

\section{Verlagerung innerhalb des Krankenhaussektors}

Innerhalb des Krankenhaussektors ist zwischen 2004 und 2005 die Verlagerung aus dem klassisch-stationären Bereich (Haupt- und Belegabteilung) in den ambulanten Bereich (ambulantes Operieren am Krankenhaus) zu erkennen. Zwischen 2005 und 2006 zeigt sich in allen Bereichen mit Ausnahme der stationären Belegabteilungen ein Anstieg. Die deutliche Steigerung in den Hauptabteilungen liegt möglicherweise daran, dass Krankenhäuser infolge der Liberalisierung des Vertragsarztrechts vermehrt nieder- 
gelassene Ärzte konsiliarärztlich in Hauptabteilungen beschäftigen, dort relativ gut honorieren und im Gegenzug Belegabteilungen schließen. Zur gesonderten Betrachtung und Förderung des Belegarztwesens wurde ein neues Kapitel in den EBM mit einer Höherbewertung der Leistungen (in Punkten) eingefügt; in Bayern fördern die Krankenkassen das Belegarztwesen zudem mit hohen Vergütungspunktwerten. Dass die ambulanten Operationen am Krankenhaus durchgängig stark ansteigen, dürfte auch an einer entsprechenden strategischen Ausrichtung der Häuser liegen. Eine weitere Ursache könnte die verstärkte Prüfung der Krankenkassen nach ambulantem Operationspotenzial sein. Trotzdem finden die meisten Krankenhausleistungen noch in den Hauptabteilungen statt: Der Anteil liegt 2006 bei 57\% (ungewichtet) bzw. 67\% (bei Berücksichtigung der Fallschwere) aller betrachteten Leistungen. Die meisten Operationen der untersuchten Indikationen finden jedoch im vertragsärztlich-ambulanten Sektor statt. Nur für die Operationen an Leisten- und Schenkelhernie sowie die rekonstruktiven arthroskopischen Operationen - zwei relativ aufwändige Prozeduren - liegt der Anteil des ambulantvertragsärztlichen Sektors mit 21\% bzw. 23\% bei weniger als der Hälfte. Am höchsten ist der Anteil bei den KataraktOperationen (79\%) und den kurativen Koloskopien (87\%).

In Tabelle 3 wird für die einzelnen Prozeduren die Veränderungsrate je Leistungssektor im Vergleich zum Vorjahr dargestellt. Für das letzte betrachtete Jahr (2006) ist zudem jeweils die Fallzahl dargestellt. ${ }^{10}$ Überraschend sind beispielsweise der Rückgang kurativer Koloskopien im vertragsärztlichen Bereich zwischen 2004 und 2005, dem kein Anstieg der präventiven Koloskopien gegenübersteht, und der starke Anstieg von Katarakt-Operationen im Krankenhaus über den gesamten Betrachtungszeitraum.

\section{Eingeschränkte Beweiskraft der Analyse und künftige Herausforderungen}

Die vorliegende Analyse ist eine der ersten Datenanalysen zur Fragestellung „,ambulant vor stationär“ für das GKV-System. Aus Gründen der Machbarkeit und Datenverfügbarkeit mussten dabei manche methodischen Einschränkungen in Kauf genommen werden, die zu einer Verzerrung der Ergebnisse führen könnten. Insbesondere dürften die Änderungen externer Rahmenbedingungen hinsichtlich des Vergütungssystems die Analyseergebnisse beeinflussen. Daneben gibt es weitere methodische Herausforderungen bei empirischen Studien zum Vergleich von Leistungen verschiedener Versorgungssettings (z. B. ambulant-stationär). Beispielsweise ist der „Goldstandard“ der ökonomischen Evaluation, die Zuordnung von Patienten nach dem Zufallsprinzip in klar voneinander abgegrenzte Interventions- und Kontrollgruppen, hier kaum umsetzbar. Außerdem ergeben sich Verzerrungen, Unsicherheiten oder Instabilitäten durch

- die Abweichung der vereinbarten Vergütungssätze von den betriebswirtschaftlichen Kosten und die dadurch induzierten Anreizreaktionen

- die aktuelle strategische und operative Ausrichtungen von Krankenhäusern (und Krankenhausträgern) zum ambulanten Bereich (z. B. im Zusammenhang mit Einrichtung von Zentren für ambulantes Operieren)

- die weit reichende Einflussnahme von Pressure-groups mit Partikularinteressen auf das Versorgungsgeschehen.

Gleichwohl wurde mit der vorliegenden Studie ein wichtiges wissenschaftliches Prinzip umgesetzt: ${ }^{11}$ Die methodischen Entscheidungen fielen vorab und ohne Kenntnis von deren Wirkung auf die Ergebnisse. Die Datengrundlage der Studie ist hinreichend groß, valide und repräsentativ. Insbesondere für den Vergleich zwischen 2005 und 2006 sind die Ergebnisse so eindeutig, dass sie zumindest als ein klarer Indikator gewertet werden können: Die beabsichtigte Leistungsverlagerung aus dem Krankenhaussektor in den - annahmegemäß eher günstigeren - ambulanten Bereich konnte im betrachteten Zeitraum nicht beobachtet werden. Die Auswertung zeigt eine Verlagerung innerhalb des Krankenhausbereichs hin zum ambulanten Operieren am Krankenhaus (vor allem zwischen 2004 und 2005) und eine massive Ausweitung der Fallzahlen in den stationären Hauptabteilungen seit 2005.

Valide Datenanalysen sind eine elementare Entscheidungsgrundlage für Verhandlungsführer in einem pluralistischen Gesundheitssystem. Bei der technischen Umsetzung der vorliegenden Datenanalyse wurde deutlich, wie wichtig konstante Rahmenbedingungen, z. B. im Vergütungssystem, und eine gute, allgemeine Datenbasis sind. Beide Voraussetzungen werden durch die jüngsten Änderungen im Gesundheitswesen eher erschwert. Obwohl erst zum 1. April 2005 mit dem EBM 2000plus ein neues 


\section{THEMA}

Tabelle 3: Veränderung je Leistungsbereich und Fallzahl 2006

\begin{tabular}{|c|c|c|c|}
\hline \multirow[t]{2}{*}{ Leistungssektor } & \multicolumn{3}{|c|}{$\begin{array}{l}\text { Veränderung in \% } \\
\text { gegenüber Vorjahr }\end{array}$} \\
\hline & 2005 & 2006 & Fallzahl 2006 \\
\hline OP Leisten-Schenkelhernie & $1,1 \%$ & $15,0 \%$ & 3.487 \\
\hline stationär Hauptabteilung & $-3,6 \%$ & $26,2 \%$ & 1.900 \\
\hline stationär Belegabteilung & $-0,8 \%$ & $-10,5 \%$ & 449 \\
\hline ambulant am Krankenhaus & $22,0 \%$ & $25,6 \%$ & 419 \\
\hline vertragsärztlich ambulant & $5,0 \%$ & $3,7 \%$ & 720 \\
\hline OP Katarakt & $6,0 \%$ & $17,1 \%$ & 4.827 \\
\hline stationär Hauptabteilung & $67,9 \%$ & $116,4 \%$ & 404 \\
\hline stationär Belegabteilung & $-7,2 \%$ & $-3,3 \%$ & 255 \\
\hline ambulant am Krankenhaus & $25,2 \%$ & $14,6 \%$ & 344 \\
\hline vertragsärztlich ambulant & $3,1 \%$ & $13,4 \%$ & 3.824 \\
\hline OP Varizen & $7,3 \%$ & $8,7 \%$ & 4.180 \\
\hline stationär Hauptabteilung & $-15,5 \%$ & $29,1 \%$ & 1.293 \\
\hline stationär Belegabteilung & $-25,2 \%$ & $-10,9 \%$ & 280 \\
\hline ambulant am Krankenhaus & $22,2 \%$ & $30,2 \%$ & 371 \\
\hline vertragsärztlich ambulant & $25,7 \%$ & $-0,4 \%$ & 2.237 \\
\hline Arthroskopische rekonstruktive OP & $6,3 \%$ & $31,0 \%$ & 2.665 \\
\hline stationär Hauptabteilung & $20,6 \%$ & $70,4 \%$ & 1.445 \\
\hline stationär Belegabteilung & $2,0 \%$ & $2,7 \%$ & 569 \\
\hline ambulant am Krankenhaus & $18,8 \%$ & $-13,0 \%$ & 46 \\
\hline vertragsärztlich ambulant & $-7,2 \%$ & $4,3 \%$ & 605 \\
\hline OP Karpaltunnel-Tarsaltunnelsyndrom & $18,1 \%$ & $3,2 \%$ & 2.495 \\
\hline stationär Hauptabteilung & $-17,6 \%$ & $26,0 \%$ & 77 \\
\hline stationär Belegabteilung & $-31,6 \%$ & $-43,4 \%$ & 15 \\
\hline ambulant am Krankenhaus & $24,1 \%$ & $14,9 \%$ & 477 \\
\hline vertragsärztlich ambulant & $19,7 \%$ & $0,6 \%$ & 1.927 \\
\hline Strahlentherapie & $12,1 \%$ & $25,8 \%$ & 2.671 \\
\hline stationär Hauptabteilung & $-3,8 \%$ & $38,5 \%$ & 887 \\
\hline stationär Belegabteilung & $-16,7 \%$ & $-3,5 \%$ & 23 \\
\hline ambulant am Krankenhaus & $0,0 \%$ & $0,0 \%$ & 0 \\
\hline vertragsärztlich ambulant & $21,5 \%$ & $20,7 \%$ & 1.761 \\
\hline Kurative Koloskopien & $-4,0 \%$ & $3,7 \%$ & 19.508 \\
\hline stationär Hauptabteilung & $-32,5 \%$ & $22,6 \%$ & 668 \\
\hline stationär Belegabteilung & $-45,3 \%$ & $67,7 \%$ & 57 \\
\hline ambulant am Krankenhaus & $23,0 \%$ & $18,7 \%$ & 1.772 \\
\hline vertragsärztlich ambulant & $-4,5 \%$ & $1,6 \%$ & 17.012 \\
\hline
\end{tabular}


Abrechnungssystem eingeführt wurde, das zudem für sich reklamierte, nach einem fast zehn Jahre andauernden und arbeitsintensiven Prozess unter Berücksichtigung internationaler Erfahrungen auf betriebswirtschaftlicher Basis entwickelt worden zu sein, erfolgte bereits zum 1. Januar 2008 eine neue EBM-Reform. Die Datenbasis erodiert durch die Ausbreitung neuer Versorgungsformen (z. B. Verträge nach §§ 140a ff SGB V), die nicht per gesetzlicher Vorgabe in dem für die Regelversorgung verpflichtenden elektronischen Datenträgeraustausch erfasst sind. Durch die Abschaffung der Bundesverbände der Kassenarten als Körperschaften besteht insbesondere für die Betriebskrankenkassen die Gefahr, dass Datenströme für eine Kassenart künftig nicht mehr an einer zentralen Datenannahmestelle zusammenfließen und nicht mehr von dort systematisch für Auswertungen im Wettbewerb der Kassenarten genutzt werden können. Allerdings könnte der Wettbewerb auch im Datenmanagement als Triebfeder zur Findung bzw. Bestätigung der für die Kassenart günstigsten Lösung wirken.

Es stellt die Krankenkassen vor eine große Herausforderung, die Versorgungsdaten sowohl aus der Regelversorgung als auch aus ihren neuen Versorgungsprojekten zusammenzuführen und somit der systematisierten Datenauswertung zugänglich zu machen. Dies gilt sowohl für die Fragestellungen des Vorher-Nachher-Vergleichs (wie in der vorliegenden Studie) wie auch für den methodisch hochwertigen Vergleich einer neuen Versorgungsform mit der parallel ablaufenden Regelversorgung. Die aktuelle Gesetzeslage erlaubt den Krankenkassen diese Zusammenführung und erleichtert zudem kassenartenübergreifende Projekte und Kooperationen. Allerdings stellt sich die Frage, ob künftig die Krankenkassen noch ausreichend Anreize für kassenartenbezogene Auswertungen haben werden. Grund hierfür ist die Neuregelung der Gesamtvergütung, die gemäß GKV-Wirtschaftlichkeitsstärkungsgesetz (GKV-WSG) ab 2009 nicht mehr kassenartenbezogen, sondern gemeinsam und einheitlich zu vereinbaren ist.

\section{Vertragliche Konsequenz der Analyse und Ausblick}

Die für Strukturverträge festgelegte Obergrenze für das Jahr 2005 wurde um ca. 4,5 Mio. € überschritten. Für das Jahr 2006 fällt die Überschreitung noch deutlicher aus und liegt bei ca. 10 Mio. $€$. Im Bereich der stationsersetzenden Leistungen ist die in diesem Beitrag präsentierte Analyse maßgeblich dafür, dass ein Großteil der Überschreitung über Vergütungsabsenkungen an die Betriebskrankenkassen zurückfließt. Details des Ausgleichs setzen die KV Bayerns und der BKK Landesverband Bayern in einer Ergänzungsvereinbarung zum Gesamtvertrag um. Für den Krankenhaussektor gibt es nur bedingt vergleichbare Instrumente, um Vertragsziele (Beitragssatzstabilität) und Fallzahl- bzw. Leistungsentwicklung auf dem Vertragswege und im Wettbewerb der Kassenarten in Einklang zu bringen. Die Ausgabenexplosion im Krankenhausbereich stellt die einzelnen Krankenkassen somit tendenziell vor noch größere Probleme als aktuell im vertragsärztlichen Sektor, zumal hier eine retrospektive Berücksichtigung der Fallzahl- und Leistungsentwicklung über die vereinbarten
Budgets hinaus durch Mehrerlösausgleiche nur gemeinsam und einheitlich zwischen den Kassenarten möglich ist.

Für die ambulant-ärztliche Versorgung wird ab 2009 das Morbiditätsrisiko von den Kassenärztlichen Vereinigungen vollständig auf die Krankenkassen übergehen. Dieser politische Schritt ist systematisch richtig, denn das Tragen des Versicherungsrisikos ist die Basisaufgabe jeder Versicherung. Allerdings muss eine solche Reformmaßnahme in einem von Informationsasymmetrien geprägten Markt wie dem Gesundheitswesen einhergehen mit der Etablierung neuer Steuerungsmechanismen. Vertragliche Konsequenzen auf regionaler Ebene, wie sie in diesem Beitrag vorgestellt wurden, sind ab $2009 \mathrm{im}$ gesamtvertraglichen Umfeld nicht mehr möglich, wohl aber im Rahmen neuer Versorgungsformen z.B. auf der Grundlage von $\$ \S 73 b$ und c SGB V. Dazu muss eine für Evaluation und Vertragscontrolling ausreichende und valide Datenbasis geschaffen werden. Für die kollektivvertragliche Vergütung wird ab 2009 die Abrechnungsprüfung an Bedeutung gewinnen.

Dass die bayerischen Strukturverträge zu stationsersetzenden Leistungen sich grundsätzlich nicht bewährt haben, kann aus der Datenanalyse nicht geschlossen werden. Die Auswertungen lassen jedoch vermuten, dass die aufgebaute Struktur im ambulant-vertragsärztlichen Bereich einen weiteren Leistungsanstieg im Krankenhaussektor nicht verhindern kann.

\section{Literatur}

AG Reha-Ökonomie im Förderschwerpunkt Rehabilitationswissenschaften (1999) [Burchert H, Hansmeier T, Hessel F, Krauth C, Nowy R, Seitz R, Wasem J]. Gesundheitsökonomische Evaluation in der Rehabilitation. Teil II: Bewertung von Ressourcenverbräuchen. DRV-Schriften; 16: 195-246.

Krauth C, Hessel F, Hansmeier T, Wasem J, Seitz R, Schweikert B (2005a) Empirische Bewertungssätze in der gesundheitsökonomischen Evaluation - ein Vorschlag der AG Methoden der gesundheitsökonomischen Evaluation (AG MEG). Das Gesundheitswesen; 67: 736-746.

Krauth C, Hessel F, Klingelhöfer HE, Schweikert B, Hansmeier T, Wasem J (2005b) Gesundheitsökonomische Evaluation von Rehabilitationsprogrammen im Förderschwerpunkt Rehabilitationswissenschaften. Rehabilitation; 44: 297-306.

Medical Tribune, 26.10.2007.

Schulenburg J-M Graf vd, Greiner W, Jost F, Klusen N, Kubin M, Leidl R, Mittendorf T, Rebscher H, Schöffski O, Vauth C, Volmer T, Wahler S, Wasem J, Weber C et al. (2007) Deutsche Empfehlungen zur gesundheitsökonomischen Evaluation - dritte und aktualisierte Fassung des Hannoveraner Konsens. Gesundheitsökonomie und Qualitätsmanagement; 12: 285-290.

Schulte G (2007) „Ambulant vor stationär“ - ein gesundheitspolitischer Grundsatz auf dem Prüfstand. In: Ulrich V,. Ried W (Hrsg.) Effizienz, Qualität und Nachhaltigkeit im Gesundheitswesen. Baden-Baden, Nomos; 517-540.

Seitz R, Krammling F-G, Lindl C (2007) „Ambulant vor stationär“ - erste empirische Ergebnisse einer BKK-Studie zu stationsersetzenden Leistungen. In: BKK Landesverband Bayern (Hrsg.) Gesundheitsversorgung zwischen Wettbewerb und Sozialstaatsprinzip - Analysen, Konzepte, Diskussionsbeiträge. München; 36-46.

Statistisches Bundesamt (2006) Grunddaten der Krankenhäuser und Vorsorge- oder Rehabilitationseinrichtungen. Fachserie 12 Reihe 6.1, Wiesbaden.

Weatherly J, Seiler R, Meyer-Lutterloh K, Schmid E, Lägel R, Amelung V (2007) Leuchtturmprojekte Integrierter Versorgung und Medizinischer Versorgungszentren. Berlin, MVV Medizinische Wissenschaftliche Verlagsgesellschaft. 


\title{
THEMA
}

\section{Fußnoten}

1 Die Autoren bedanken sich bei den Mitgliedern der Arbeitsgruppe Vertragsärztliche Vergütung von der Audi BKK, BKK BMW, BKK Mobil Oil, HypoVereinsbank BKK, PBK sowie SBK für ihre Unterstützung bei der Durchführung des Projekts. Für wertvolle Kommentare zum Skript wie auch die Entwicklung eines großen Teils der Abfragen geht ein herzlicher Dank an unsere Kollegin Eva Adler. Den Kollegen aus der Abteilung Servicemanagement danken wir für die Bereitstellung der Daten zur amtlichen Statistik und ihre Unterstützung bei deren Interpretation.

2 Bei den über 4.000 IGV-Verträgen stehen mit 25\% Knie- und Hüftendoprothesen an der Spitze der Indikationsliste. „Ich wüsste nicht, was es da zu integrieren gibt“, wird der Vorsitzende des Sachverständigenrats, Prof. Wille dazu zitiert (Medical Tribune, 26.10.2007; S. 25). Selten sind dagegen mit 0,04\% psychosomatische Erkrankungen.

3 AG Reha-Ökonomie (1999). Die Arbeitsgruppe hat sich 2005 umbenannt in AG MEG: Arbeitsgruppe Methoden der Gesundheitsökonomischen Evaluation (Krauth et al. 2005a).

4 Dies gilt zumindest für alle stationären Behandlungsfälle, bei denen aus medizinischen Gründen eine Entlassung aus dem Krankenhaus vor dem Erreichen der unteren Grenzverweildauer, deren Unterschreiten zu tagesbezogenen Abschlägen bei der Pauschalvergütung führt, nicht möglich ist.

5 Wurde vor Einführung der DRGs beispielsweise ein Patient mit einer internistischen Erkrankung aufgenommen und fand sich auch ein chirurgisches Problem (z. B. Leistenhernie), so wurde der Patient im Allgemeinen nach Lösung des internistischen Problems während desselben Krankenhausaufenthalts operiert. Unter aktuellen DRG-Bedingungen wird er tendenziell nach Behandlung des internistischen Problems entlassen und die Leistenhernie einige Wochen später operiert.

6 Diesem Rückgang der Bettenzahl steht ein starker Ausgabenanstieg für häusliche Krankenpflege gegenüber: Mit einer Verdreifachung (Indexwert: $336 \%$ ) weist dieser Bereich den höchsten Anstieg auf, wird aber wegen der relativ niedrigen quantitativen Bedeutung (2,12 Mrd. € im Jahr 2006) nicht in Abb. 1 aufgeführt

7 Für den Bereich Katarakt wurde die Fallzahlanalyse durch eine Kostenschätzung ergänzt (Vergleich des vertragsärztlich-ambulanten Bereichs mit Krankenhauskosten). Diese ergab folgendes Ergebnis: Eine stationäre Kataraktoperation ist im Schnitt (Median: $1.280 €$; Mittelwert: 1.360 $€)$ über $200 €$ teurer als die ambulante Operation im vertragsärztlichen Bereich (Median und Mittelwert ca. 1.050 €). Die stationäre Kataraktoperation ist jedoch ca. $150 €$ günstiger, wenn sie an einem Belegtag durchgeführt wird. Die stationären Ein-Tages-Fälle sind aber sehr selten und aus finanzieller Perspektive des Krankenhauses nicht sinnvoll.

8 Bei einer Überschreitung dieser Vergleichsgröße finden zwischen den Gesamtvertragspartnern (BKK Landesverband und KV Bayerns) weitere gezielte Analysen der einzelnen Leistungsbereiche statt, die nicht Gegenstand dieses Beitrags sind.

9 Für das Jahr 2006 wurden insgesamt, d. h. ohne Berücksichtigung von Mitglieder- und Kostengewichtung, 40.684 Fälle analysiert. Bei den Abfragen für das Jahr 2005 im Krankenhausbereich weichen die Ergebnisse der Abfragen 2004/2005 und 2005/2006 leicht voneinande ab. Dies dürfte in erster Linie an der Änderung der DRGs für einzelne Leistungsbereiche und an der Zeitverzögerung der Abrechnung liegen. In der Summe ist diese Abweichung jedoch untererheblich: Bei den ungewichteten Fallzahlen liefert die zweite Abfrage für 2005 vierzehn weniger Fälle als die erste (9.439 bzw. 9.425), was einem Anteil von 0,15\% entspricht. Bei Mitglieder- und Kostengewichtung der Ergebnisse ergeben sich 31 bzw. 0,44\% zusätzliche Fälle. In den Tabellen sind die Ergebnisse für die aktuellere Abfrage dargestellt.

10 Die detaillierten Ergebnisse des Vergleichs 2005 zu 2004 sind in Seitz/ Krammling/Lindl (2007) dargestellt.

11 Als Orientierung dienten die Empfehlungen der AG Reha-Ökonomie bzw. AG MEG (Krauth et al. 2005) und der Hannoveraner Konsens (aktuell: Schulenburg et al. 2007).

\section{Der „Klassiker“ - noch immer konkurrenzlos.}

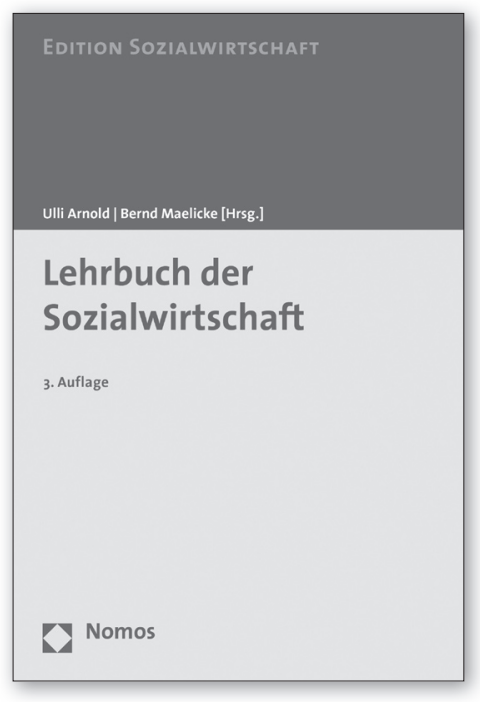

\author{
Lehrbuch der Sozialwirtschaft \\ Von Prof. Dr. Dr. h.c. Ulli Arnold \\ und Prof. Dr. Bernd Maelicke \\ 3. Auflage 2008, ca. 600 S., brosch., ca. 39,- $€$, \\ ISBN 978-3-8329-2680-9 \\ (Edition Sozialwirtschaft, Bd. 9) \\ Erscheint August 2008
}

Die rapide Veränderung der politischen und strukturellen Rahmenbedingungen für die Sozialwirtschaft erfordert grundlegende Änderungen in der Ausbildung und Weiterbildung von Führungskräften sozialer Dienstleistungsorganisationen. Die vollständig aktualisierte und neu bearbeitete 3. Auflage vermittelt Basiswissen und aktuelle Informationen zu den wichtigsten Themen. 